\title{
Real-space renormalisation group approach to driven diffusive systems
}

\author{
T. Hanney ${ }^{1}$ and R. B. Stinchcombe ${ }^{2}$ \\ ${ }^{1}$ SUPA and School of Physics, University of Edinburgh, Mayfield Road, \\ Edinburgh, EH9 3JZ, UK \\ ${ }^{2}$ Theoretical Physics, 1 Keble Road, Oxford, OX1 3NP, UK
}

\begin{abstract}
We introduce a real-space renormalisation group procedure for driven diffusive systems which predicts both steady state and dynamic properties. We apply the method to the boundary driven asymmetric simple exclusion process and recover exact results for the steady state phase diagram, as well as the crossovers in the relaxation dynamics for each phase.
\end{abstract}

\section{Introduction}

Nonequilibrium phenomena are observed throughout nature and society, and can often be thought of as some kind of driven diffusive system [1]. Examples include chemical kinetics, the motion of interacting molecular motors along a substrate, population dynamics in an ecology, the exchange of wealth or commodities in an economy, traffic flow and many more. The ongoing interest in driven systems has been sustained by the wide variety of behaviour the model systems exhibit, which incorporate not only elements familiar from equilibrium statistical mechanics, such as phase transitions, cooperative behaviour, and so on, but also many dramatic new features. These include phase transitions induced by external driving and even in one dimension; long-range (i.e. power-law) correlations, not only at criticality, but generically in the steady state; and very rich dynamics.

Model driven diffusive systems are typically defined on a lattice on which particles hop from site to site, where the precise definition of the stochastic particle dynamics is motivated on physical grounds. Nonequilibrium steady states are constructed by driving a current of particles through the system. Despite being simply stated, they still exhibit the wide range of phenomena. Indeed, a virtue of their simplicity is that in some cases the models can be solved exactly. However, most cases elude exact solution; moreover, even in cases where the steady state can be written down, the task of computing macroscopic observables may be too difficult. For these reasons, it is necessary to develop approximate techniques.

A technique that has been hugely successful in equilibrium statistical mechanics is the renormalisation group procedure. Here, we show how one can adapt these equilibrium methods to nonequilibrium systems, where we devise a way to accommodate the feature that distinguishes the nonequilibrium steady state from its equilibrium counterpart - the existence of currents - in the renormalisation group prescription. In particular, our approach includes the effects of fluctuations, which, in certain equilibrium and nonequilibrium systems, destroy the transitions predicted 


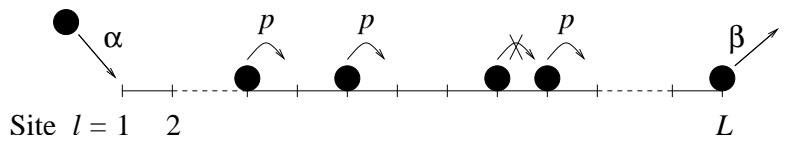

Figure 1. Dynamics of the ASEP with particle injection at the left boundary and removal from the right boundary

by mean field theory. Thus our approach builds on previous prescriptions [2], and it complements real-space techniques devised for quantum-spin chains, and adapted to stochastic models [3, 4].

We test our approach using the asymmetric simple exclusion process with open boundaries, which we introduce and review in section 2 In section 3 we outline the matrix product formulation of the master equation. This formulation provides a convenient notation for the purposes of the steady state renormalisation which we describe in section 4 We recover the exact critical point and find qualitative agreement for the phase diagram. We extend the renormalisation to the dynamics in section 5 and again we obtain qualitative agreement with the exact predictions for the relaxation dynamics.

\section{The asymmetric simple exclusion process (ASEP)}

The asymmetric simple exclusion process with open boundaries is one of the most widely studied driven diffusive systems. Exact results show that it undergoes both first- and second order phase transitions in the steady state and exhibits different relaxation dynamics in each of its phases. For these reasons, it represents an ideal test bed with which to assess the efficacy of approximate methods.

The model is defined on a chain of $L$ sites, labelled $l=1, \ldots, L$. The occupation variable $n_{l}=0$ if site $l$ is vacant and $n_{l}=1$ if it is occupied; multiple occupancy is forbidden. The stochastic dynamics are illustrated in figure 1 in continuous time, a particle at site $l<L$ hops to its nearest neighbour site to the right with rate $p$, provided it is empty. If site 1 is vacant, it becomes occupied with rate $\alpha$, and if there is a particle at site $L$, it leaves the system with rate $\beta$.

The phase diagram for this model is known exactly [5]. In the limit of large $L$, depending on the values of the ratios $\alpha / p$ and $\beta / p$, the system may reside in one of three possible phases (see figure 2): for $\alpha / p<1 / 2$ and $\alpha<\beta$, the system is in a low density phase with particle density $\rho<1 / 2$; for $\beta / p<1 / 2$ and $\alpha>\beta$, the system is in a high density phase, $\rho>1 / 2$; for $\alpha / p>1 / 2$ and $\beta / p>1 / 2$ the current, $J$,through the system assumes its maximum value $J=1 / 4$ and the density $\rho=1 / 2$. The transition between the high and low density phases, indicated by the dotted line in the phase diagram, is first order - along the transition line a low density region coexists with a high density region and the system density $\rho=1 / 2$. The transition between these low current phases and the maximum current phase, indicated by the solid line in the phase diagram, is second order.

The relaxation dynamics have also been obtained recently using the Bethe ansatz [6]: in the maximum current phase the relaxation time scales as $L^{z}$ where the dynamic exponent $z=3 / 2$; along the coexistence line $z=2$; in the two low current phases away from the coexistence line, the relaxation times are finite. 


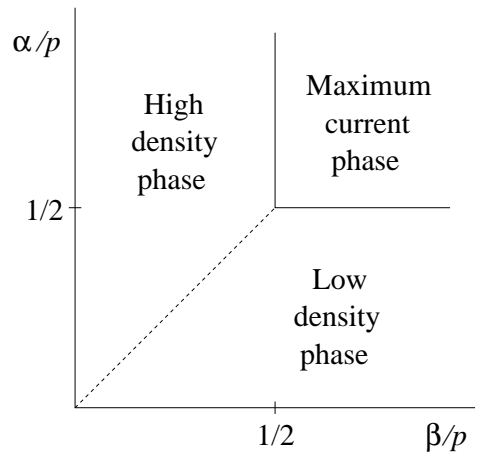

Figure 2. Exact phase diagram for the boundary driven ASEP.

\section{Matrix product formulation of the ASEP}

The exact solution for the steady state of the ASEP was obtained using a matrix product technique [5]. In this approach, the aim is to represent the probability that one observes the system in a configuration $\underline{n}=\left(n_{1}, \ldots, n_{L}\right)$ at time $t, P(\underline{n}, t)$, as

$$
P(\underline{n}, t)=Z^{-1}\left\langle W\left|\prod_{l=1}^{L}\left(n_{l} D+\left(1-n_{l}\right) E\right)\right| V\right\rangle,
$$

where $D$ and $E$ are (in general time-dependent) matrices, and $\langle W|$ and $|V\rangle$ are (timeindependent) vectors. Here, $Z$ is a normalisation given by

$$
Z=\left\langle W\left|C^{L}\right| V\right\rangle \text {. }
$$

where we have introduced the (time-independent) matrix $C=D+E$. Thus, a string of matrices is used to represent a configuration of the particles in the system, where the matrix $D$ is used to represent an occupied site and $E$ a vacant site.

\subsection{Steady state algebra}

In the limit $t \rightarrow \infty$, it was shown in [5] that (1) and (2) do indeed give the steady state probabilities provided the matrices $D$ and $E$ and the vectors $\langle W|$ and $|V\rangle$ satisfy the algebra

$$
\begin{aligned}
& D+E=D E, \\
& \langle W| E=(p / \alpha)\langle W|, \\
& D|V\rangle=(p / \beta)|V\rangle .
\end{aligned}
$$

Moreover, matrix representations were found which satisfy (31)-(51). We remark however that in order to compute the probabilities to observe a particular configuration, it is not necessary to find such matrix representations; it is instead sufficient to use (3) to order all the matrices $E$ to the left of the matrices $D$, and then use the boundary conditions (4) and (51) to obtain probabilities in terms of $\alpha, \beta$ and $p$.

\subsection{Dynamic algebra}

In [7, it was shown how the steady state algebra can be generalised to a full dynamic algebra such that (11) and (2) represent the probabilities at all times $t$. In this case, 


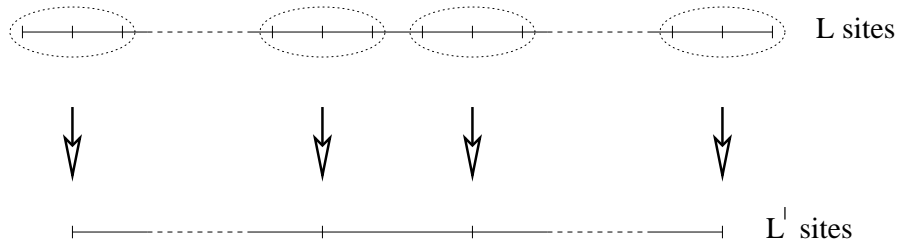

Figure 3. Blocking the lattice into groups of $b=3$ sites.

the matrices and vectors must satisfy

$$
\begin{aligned}
& \dot{D}=\left[C^{-1}, \Lambda\right], \\
& \Lambda C^{-1} D=D C^{-1} \Lambda, \\
& \langle W|\left(\alpha / p-C^{-1} \Lambda\right)=0, \\
& \left(\beta / p-\Lambda C^{-1}|V\rangle=0,\right.
\end{aligned}
$$

where $\Lambda=D E$ is a current operator (capturing the fact that in order for a particle to hop there must be a particle to the left of a vacancy).

\section{Steady state renormalisation of the ASEP}

The key feature which distinguishes nonequilibrium steady states from equilibrium ones is the presence of currents. Moreover, in driven diffusive systems with open boundaries, the boundary dynamics determine the particle current and density throughout the bulk of the system. This is in marked contrast to equilibrium systems where boundary effects are typically localised to the vicinity of the boundary.

In this section, we present a renormalisation group method which accounts for these currents by employing a blocking procedure designed to accommodate the influence of the boundaries throughout the bulk of the system.

\subsection{Blocking transformation}

A blocking transformation is implemented by grouping sites of the lattice into blocks where each block contains $b$ sites, as illustrated for the case $b=3$ in figure 3 These blocks are then identified with the sites of a renormalised lattice with a number of sites $L^{\prime}=L / b$. This must be supplemented by a prescription which identifies particle configurations within a block with a single coarse-grained block variable. We achieve this by using a majority rule identification, which for $b=3$ is

$$
\begin{aligned}
& D^{\prime}=D D D+D D E+D E D+E D D, \\
& E^{\prime}=E E E+E E D+E D E+D E E .
\end{aligned}
$$

The issue for any such renormalisation group treatment then is what quantities are held fixed under the blocking and which are rescaled. We keep the current across each bond $J$ and the bulk density $\rho$ fixed, and rescale the parameters $\alpha / p$ and $\beta / p$. To achieve this, we consider pairs of adjacent blocks separately, and replace the effect of particle exchange with the rest of the system with particle injection and ejection at the boundaries of the pair of blocks, with rates $\alpha / p$ and $\beta / p$, as illustrated in figure 4. Thus the current and density in the rescaled system are written 


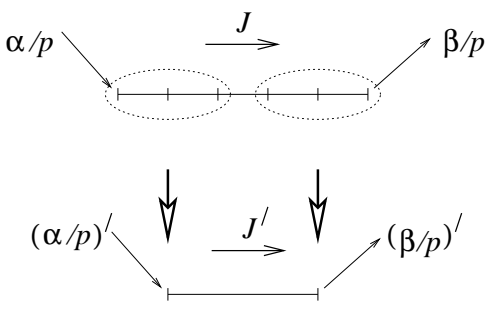

Figure 4. Blocking transformation at fixed current for the boundary driven ASEP with $b=3$.

$$
\begin{aligned}
& J^{\prime}=\frac{\left\langle W^{\prime}\left|D^{\prime} E^{\prime}\right| V^{\prime}\right\rangle}{\left\langle W^{\prime}\left|C^{\prime 2}\right| V^{\prime}\right\rangle}, \\
& \rho^{\prime}=\frac{\left\langle W^{\prime}\left|D^{\prime}\right| V^{\prime}\right\rangle}{\left\langle W^{\prime}\left|C^{\prime}\right| V^{\prime}\right\rangle} .
\end{aligned}
$$

Under the majority rule matching between configurations, (10), (11), the block current $J$ and the block density $\rho$ assume the form

$$
\begin{aligned}
& J=\frac{\left\langle W\left|\left(D^{3}+D^{2} E+D E D+E D^{2}\right)\left(E^{3}+E^{2} D+E D E+D E^{2}\right)\right| V\right\rangle}{\left\langle W\left|C^{6}\right| V\right\rangle}, \\
& \rho=\frac{\left\langle W\left|\left(D^{3}+D^{2} E+D E D+E D^{2}\right)\right| V\right\rangle}{\left\langle W\left|C^{3}\right| V\right\rangle} .
\end{aligned}
$$

Note that we impose that neither the vectors and $\langle W|$ and $|V\rangle$ nor the matrix algebra (3) to (57) are changed under the rescaling. This reflects the fact that both the scaled and the unscaled configurations are taken to evolve under the same stochastic dynamics: any extension of the parameter space is prohibited under the rescaling. Finally, the rescaling of the parameters $\alpha / p$ and $\beta / p$ is achieved by setting $J^{\prime}=J$ and $\rho^{\prime}=\rho$.

\section{2. $\alpha=\beta$}

Up to this point, the use of the matrix product formulation has been nothing more than a choice of notation. The task now is to evaluate the correlation functions (12) to (15) and this is relatively straightforward if one makes use of the algebra (31) to (5), though we remark that even here the algebra only simplifies the calculations: one could evaluate the relevant correlations directly from the master equation, for example. For the case $\alpha=\beta$ one obtains (still for $b=3$ )

$$
\rho^{\prime}=\rho=1 / 2,
$$

which is also the result obtained in the exact solution for $\alpha=\beta$, and

$$
\frac{2 x^{\prime}}{3 x^{\prime 2}+2 x^{\prime}}=\frac{66 x+83 x^{2}+46 x^{3}+12 x^{4}}{84 x+126 x^{2}+112 x^{3}+70 x^{4}+30 x^{5}+7 x^{6}},
$$

from matching $J^{\prime}=J$, where $x=p / \alpha$. Stable fixed points of this equation, $(\alpha / p)^{*}=(\alpha / p)=(\alpha / p)^{\prime}$, are found at $(\alpha / p)^{*}=0$ and $(\alpha / p)^{*}=3.959$, and these are separated by an unstable fixed point at $(\alpha / p)^{*}=1 / 2$. At the unstable fixed point, $J^{*}=1 / 4$. Thus the $(\alpha / p)^{*}=0$ fixed point represents the low-current phase, and the transition between this phase and the maximum current phase is given by the unstable 


\begin{tabular}{|c|c|c|c|c|c|c|c|c|c|c|c|}
\hline & $\mathrm{A}$ & $\mathrm{B}$ & $\mathrm{C}$ & $\mathrm{D}$ & $\mathrm{E}$ & $\mathrm{F}$ & $\mathrm{G}$ & & & & \\
\hline$(\alpha / p)^{*}$ & $\frac{1}{2}$ & 1 & 0 & 0 & 3.248 & 0.423 & 3.959 & 0.415 & $\infty$ & 2.795 & $\infty$ \\
$(\beta / p)^{*}$ & $\frac{1}{2}$ & 0 & 1 & 0 & 0.423 & 3.248 & 3.959 & $\infty$ & 0.415 & $\infty$ & 2.795 \\
\hline
\end{tabular}

Table 1. Fixed points of the steady state blocking transformation for $b=3$. The values given as decimals are evaluated numerically — all the rest are determined analytically. The fixed points depicted in the flow diagram shown in figure 5 are labelled A to $\mathrm{G}$.

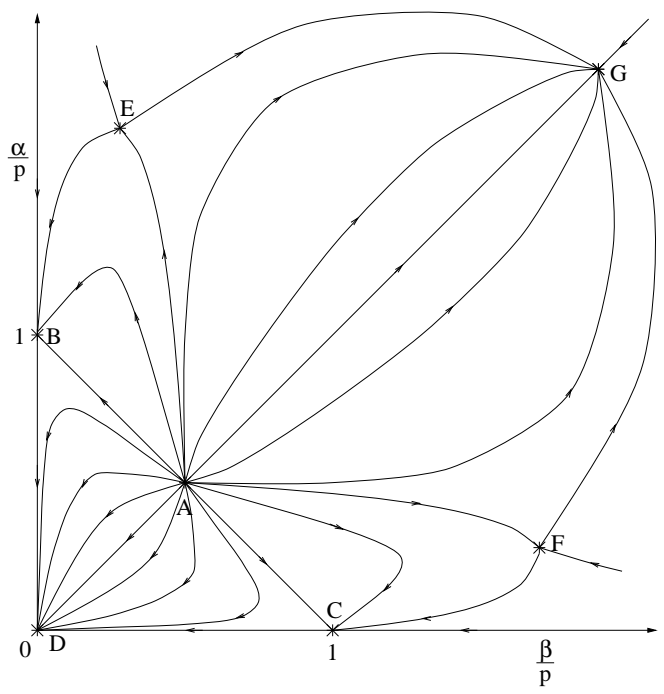

Figure 5. Schematic flow diagram in the $\alpha / p-\beta / p$ plane for generic values of $b$. The locations of the the fixed points $\mathrm{A}$ to $\mathrm{D}$ do not depend on $b$, the remaining fixed point values are $b$-dependent.

fixed point, for which we recover the exact value $(\alpha / p)^{*}=1 / 2$. Moreover the current and density at this point are also given by their exactly known values throughout the maximum current phase: $J^{*}=1 / 4$ and $\rho^{*}=1 / 2$.

\section{3. $\alpha \neq \beta$}

For $\alpha \neq \beta$ the scaling equations are straightforward to obtain, but rather lengthy so we do not include them here. Their fixed point structure is given in table 1 It is also straightforward to obtain the flow diagram by making some choice for an initial set of parameters and seeing how they evolve under several iterations of the scaling transformation. The result is given in figure [5] and should be compared with the exact phase diagram shown in figure 2

The fully unstable fixed point A controls the second-order transition between the low current and maximum current phases. At this point, the current and density assume their exact critical values $\left(\rho_{c}, J_{c}\right)=\left(\frac{1}{2}, \frac{1}{4}\right)$. The flow line AE is a separatrix which marks the boundary between the high density regime in the low current phase (represented by point B) and the maximum current phase. Similarly, the line AF separates the low density regime in the low current phase (represented by point $\mathrm{C}$ ) from the maximum current phase. Points B, C and D are all zero current fixed points. 
The first-order transition in the model is represented by the flow line $\mathrm{AD}$, which separates (exactly) the high density from the low density regimes in the low current phase. Also, across the line $\alpha / p+\beta / p=1$, the gradient of the density profile near the boundary changes sign. This is the line BAC in the flow diagram.

Thus we have interpretations for the fixed points A to D. For the remaining fixed points, we have no such physical interpretation, though their presence is necessary for the flow diagram to adopt the same qualitative structure as the phase diagram. Critical exponents can be obtained by linearising the scaling equations in the vicinity of the critical fixed point. Also, the scaling outlined above was illustrated for blocking six sites to two $(b=3)$. It is also possible to perform the rescaling for eight sites to two $(b=4)$ or nine sites to three $(b=3)$, for example, thereby increasing the block size or the size of the renormalised clusters. Though these considerations do not effect the topology of the phase diagram, they may improve predictions for exponents. We illustrate this point more fully, for dynamic scaling, in the next section.

\section{Dynamic scaling for the ASEP}

So far, the scaling has concerned only the statics. In this section, we present a blocking transformation in order to learn about the relaxation to the steady state. The blocking proceeds in the same spirit as in the previous section and exploits the dynamic algebra (6) to (91). Then the scaling transformation, linearised in the vicinity of the physical steady state fixed points (A to D in the flow diagram), informs us of the nature of the relaxation dynamics in each of the corresponding phases.

\subsection{Blocking transformation}

We begin by replacing blocks containing two sites with a single renormalised site (and will generalise subsequently). This single site is parameterised by $(\alpha / p)^{\prime}$ and $(\beta / p)^{\prime}$ and its dynamics are governed by the time evolution of $\left\langle D^{\prime}(t)\right\rangle$ :

$$
\frac{\partial\left\langle D^{\prime}(t)\right\rangle}{\partial t}=\frac{\left\langle W^{\prime}\left|\dot{D}^{\prime}(t)\right| V^{\prime}\right\rangle}{\left\langle W^{\prime}\left|C^{\prime}\right| V^{\prime}\right\rangle}
$$

(c.f. equation (13)). Again, the coarse-grained block variable is identified with configurations in the original system through a majority rule statement which, for blocks containing two sites, assumes the form

$$
D^{\prime}=D D+\frac{1}{2}(D E+E D)=\frac{1}{2}(D C+C D) .
$$

This, carried into (18), yields the evolution in the blocked system:

$$
\frac{\partial\left\langle D^{\prime}(t)\right\rangle}{\partial t}=\frac{\langle W|[\dot{D}(t) C+C \dot{D}(t)]| V\rangle}{2\left\langle W\left|C^{2}\right| V\right\rangle} .
$$

Now the dynamic algebra is used to compute the time evolution of the relevant correlation functions. Although the rhs of (20) depends only on $\langle D C\rangle$ and $\langle C D\rangle$, the evolution of these two correlation functions depends on the third time-dependent function $\langle D D\rangle$. Thus the time evolution of the two-site block can be expressed as a matrix equation, where the relaxation time is determined by the largest eigenvalue of a $3 \times 3$ matrix. This eigenvalue gives the gap to the steady state eigenvalue (which is zero), and we refer to this gap as $\Delta^{\left(N_{c}\right)}$, where $N_{c}$ represents the number of sites in the block: here, $N_{c}=2$. 
Now, under the coarse-graining, we hold the gap fixed therefore we set

$$
\Delta^{\left(N_{c}^{\prime}\right)}=\Delta^{\left(N_{c}\right)},
$$

where $\Delta^{\left(N_{c}^{\prime}\right)}$ is the gap in the blocked system and $N_{c}^{\prime}$ is the number of sites in the coarse-grained system: here, $N_{c}^{\prime}=1$. Thus the dilation factor $b=N_{c}^{\prime} / N_{c}$. Through equation (21) we obtain a scaling equation in terms of the boundary parameters. Because only a single quantity - the gap — is fixed under scaling, we can derive a scaling relationship for a single parameter only. Thus we consider the case $\alpha_{p}=\beta_{p}$. This is sufficient to probe the dynamics near the steady state fixed points A and D. The scaling relationship between $(\alpha / p)^{\prime}$ and $\alpha / p$ is not in general linear, therefore it is possible that the dynamics at the zero-current fixed point and those at the critical fixed point are characterised by different values of the dynamic exponent $z$. Linearising the scaling equation near either of these fixed points obtains

$$
\alpha_{p}^{\prime}=\lambda \alpha_{p}
$$

which for each fixed point specifies the associated eigenvalue and exponent $\lambda=b^{-z}$.

\section{2. $\alpha=\beta$}

We now illustrate this procedure for $N_{c}=2$ and $N_{c}^{\prime}=1$ in more detail, in the case $\alpha=\beta$. The dynamic algebra (6) to (9) applied to equation (18) yields

$$
\langle\dot{D}(t)\rangle=(\alpha / p)^{\prime}-2(\alpha / p)^{\prime}\langle D(t)\rangle,
$$

and the gap is easily seen to be $\Delta^{(1)}=2(\alpha / p)^{\prime}$ where the prime is used to indicate a parameter in the coarse-grained system. For the two-site system, the dynamic algebra applied to (20) leads to the evolution of the correlation functions which can be represented as

$$
\partial_{t}\left(\begin{array}{c}
\langle D D\rangle \\
\langle D C\rangle \\
\langle C D\rangle
\end{array}\right)=\left(\begin{array}{ccc}
-2 \alpha / p & 0 & \alpha / p \\
1 & -\left(1+\alpha_{/}\right) & 0 \\
-1 & 1 & -\alpha / p
\end{array}\right)\left(\begin{array}{c}
\langle D D\rangle \\
\langle D C\rangle \\
\langle C D\rangle
\end{array}\right)
$$

from which the gap is found to be $\Delta^{(1)}=\alpha / p$. Thus we obtain the scaling equation

$$
(\alpha / p)^{\prime}=\frac{\alpha}{2 p}
$$

therefore $\lambda=1 / 2$ so $z=1$. We remark again that one could just as easily perform this scaling working directly with the master equation: the operator algebra simply provides a neat formulation for our purposes.

In this case the same dynamic exponent is obtained for zero-current and critical fixed points, but this is peculiar to blocking from two sites to one. In other cases, one obtains for $(\alpha / p)^{\prime}$ a complicated nonlinear function of $\alpha / p$. This function is linearised in the vicinity of the steady state fixed points $\mathrm{A}$ and $\mathrm{D}$ in order to compute the dynamic exponent in the phases they represent. The results of such a linearisation, for several values of $N_{c}$ and $N_{c}^{\prime}$ are shown in table 2. We also show the exact values which have only recently been obtained [6]. Our results appear to capture the crossover from $z=3 / 2$ dynamics in the maximum current phase (represented by point A) to $z=2$ dynamics along the coexistence line (represented by point $\mathrm{D}$ ). 


\begin{tabular}{|c|c|c|}
\hline$N_{c} \rightarrow N_{c}^{\prime}$ & $z$ at $\alpha / p=\beta / p=0$ & $z$ at $\alpha / p=\beta / p=1 / 2$ \\
\hline $2 \rightarrow 1$ & 1 & 1 \\
\hline $3 \rightarrow 1$ & 1.118 & 1.072 \\
$3 \rightarrow 2$ & 1.319 & 1.196 \\
\hline $4 \rightarrow 1$ & 1.194 & 1.111 \\
$4 \rightarrow 2$ & 1.388 & 1.221 \\
$4 \rightarrow 3$ & 1.486 & 1.257 \\
\hline $5 \rightarrow 1$ & 1.249 & 1.133 \\
$5 \rightarrow 2$ & 1.437 & 1.234 \\
$5 \rightarrow 3$ & 1.531 & 1.265 \\
$5 \rightarrow 4$ & 1.589 & 1.274 \\
\hline Exact & 2 & $3 / 2$ \\
\hline
\end{tabular}

Table 2. Estimates of the dynamic exponent near the zero current and $J=1 / 4$ fixed points (points A and D in Figure 5).

\section{3. $\beta=1-\alpha$}

One can also obtain scaling equations for the case $\beta=1-\alpha$ in order to probe the dynamics at points $\mathrm{B}$ and $\mathrm{C}$ in the flow diagram, points which represent the high and low density phases, respectively. One finds that at both B and $\mathrm{C},(\alpha / p)^{\prime}=(\alpha / p)$ for all $N_{c}$ and $N_{c}^{\prime}$. This implies there is no system size dependence in the leading contribution to the gap for large $L$, which is consistent with the exact prediction of finite relaxation times in the low current phases away from the coexistence line [6].

\section{Conclusion}

We have developed a renormalisation group procedure which we have applied to the boundary driven asymmetric simple exclusion process. In the steady state transformation, exact results for the critical point are recovered as well as a flow diagram that shares the qualitative structure of the exact phase diagram. The dynamic scaling also captures the crossovers in the exactly computed relaxation dynamics in each phase of the ASEP.

These scaling methods seem to be widely applicable: they can be generalised to higher dimensions, multiple particle species and multiple site occupancy. Since only small clusters give, through the scaling, even the highly correlated properties, the scaling does not depend on the availability of exact results, and may be particularly useful in cases where the exact steady state can be written down but any calculation of macroscopic properties remains difficult. They are also direct and straightforward to interpret, without requiring any translation from quantum spins or abstract fields as in field-theoretic methods.

\section{Acknowledgments}

T. H. would like to thank the SUPA and the EPSRC for support under programme grant GR/S10377/01. 
Real-space renormalisation group approach to driven diffusive systems

\section{References}

[1] B. Schmittmann and R. K. P. Zia, Statistical Mechanics of Driven Diffusive Systems (Academic Press, New York, 1995)

[2] I. T. Geogiev and S. R. MacKay, Phys. Rev. E 67, 056103 (2003)

[3] J. Hooyberghs and C. Vanderzande, J. Phys. A 33, 907 (2000); J. Hooyberghs and C. Vanderzande, Phys. Rev. E 63, 041109 (2001)

[4] T. Hanney and R. B. Stinchcombe, Phys. Rev. E 72, 016118 (2005)

[5] B. Derrida, M. R. Evans, V. Hakim and V. Pasquier, J. Phys. A 26, 1493 (1992)

[6] J. de Gier and F. H. L. Essler, Phys. Rev. Lett. 95, 240601 (2005)

[7] R. B. Stinchcombe and G. M. Schütz, Europhys. Lett. 29, 663 (1995) 